\title{
Classification of B Cell Subsets Based on their Cytokine Production Capacity
}

\author{
Ayumi Yoshizaki* \\ Department of Dermatology, The University of Tokyo Graduate School of Medicine, Tokyo, Japan
}

*Corresponding author: Ayumi Yoshizaki, Department of Dermatology, The University of Tokyo Graduate School of Medicine, 7-3-1 Hongo, Bunkyo-ku, 113-8655, Tokyo, Japan

ARTICLE INFO

Received: 幽 June 06, 2020

Published: 㓞 June 18, 2020

Citation: Ayumi Yoshizaki. Classification of B Cell Subsets Based on their Cytokine Production Capacity. Biomed J Sci \& Tech Res 28(2)-2020. BJSTR. MS.ID.004638.
ABSTRACT

B cells have long been regarded as cells specialized in antibody production. However, recent studies have shown that the roles of B cells are diverse. One of their key functions is that $\mathrm{B}$ cells are involved in the regulation of the immune system by producing cytokines. In fact, B cell depletion therapy is effective in a variety of autoimmune diseases, indicating that B cells also play a central role in autoimmune diseases. The B cells are composed of various subsets. Because the function of each B cell subset differs from one another, it is important to understand the role of each in the autoimmune disease pathogenesis. Recently, B cells have been shown to be classified by their producing cytokines. This article outlined B cell subsets based on the cytokines they produce.

Keywords: B Cell Subset; Effector B Cell; Regulatory B Cell; Cytokine-Producing B Cell; Autoimmune Disease

\section{Introduction}

Recent advances in immunology have revealed that B cells have a variety of functions [1]. Although originally thought to be solely responsible for producing antibodies, it has now been suggested that B cells play a pivotal role in the immune system via antigen presentation and cytokine production (Figure 1). B cells are also important in autoimmune diseases, and in particular, B cell receptor-mediated auto antigen stimulation may induce activation of auto antigen-reactive B cells and cytokine production from them, which may play a major role in the disease development. However, due to technical difficulties, direct examination of autoantigenreactive $\mathrm{B}$ cells has not yet been carried out, leaving their function unclear [2]. Nevertheless, the importance of B cells in autoimmune diseases is evident from the fact that B cell depletion therapy is useful for many systemic autoimmune diseases such as vasculitis, rheumatoid arthritis, systemic lupus erythematosus, and systemic sclerosis [3]. Since the therapeutic effect of B cell depletion is not necessarily exerted by lowering the titer of auto antibodies, it is strongly suggested that B cells themselves affect autoimmune diseases in an antibody-producing ability-independent manner. More specifically, it is hypothesized that B cells can influence the function of other immune cells, including $\mathrm{T}$ cells, by producing cytokines [4]. This article summarized three major B cell subsets that are identified by cytokine production.

\section{B Cell Subsets}

Recent studies have indicated that there are two types of B cell classification: the subsets divided by differentiation stage and the functional subsets divided by the cytokines they produce [5]. Functional B cell subsets have been shown to exist as subsets of effector B (Be) cells that activate immunity and regulatory B (Breg) cells that suppress immunity (Figure 2). Furthermore, Be cells can be divided into Be-1 cells that produce $\mathrm{T}$ helper (Th)1-type cytokines and Be-2 cells that produce Th2-type cytokines. 


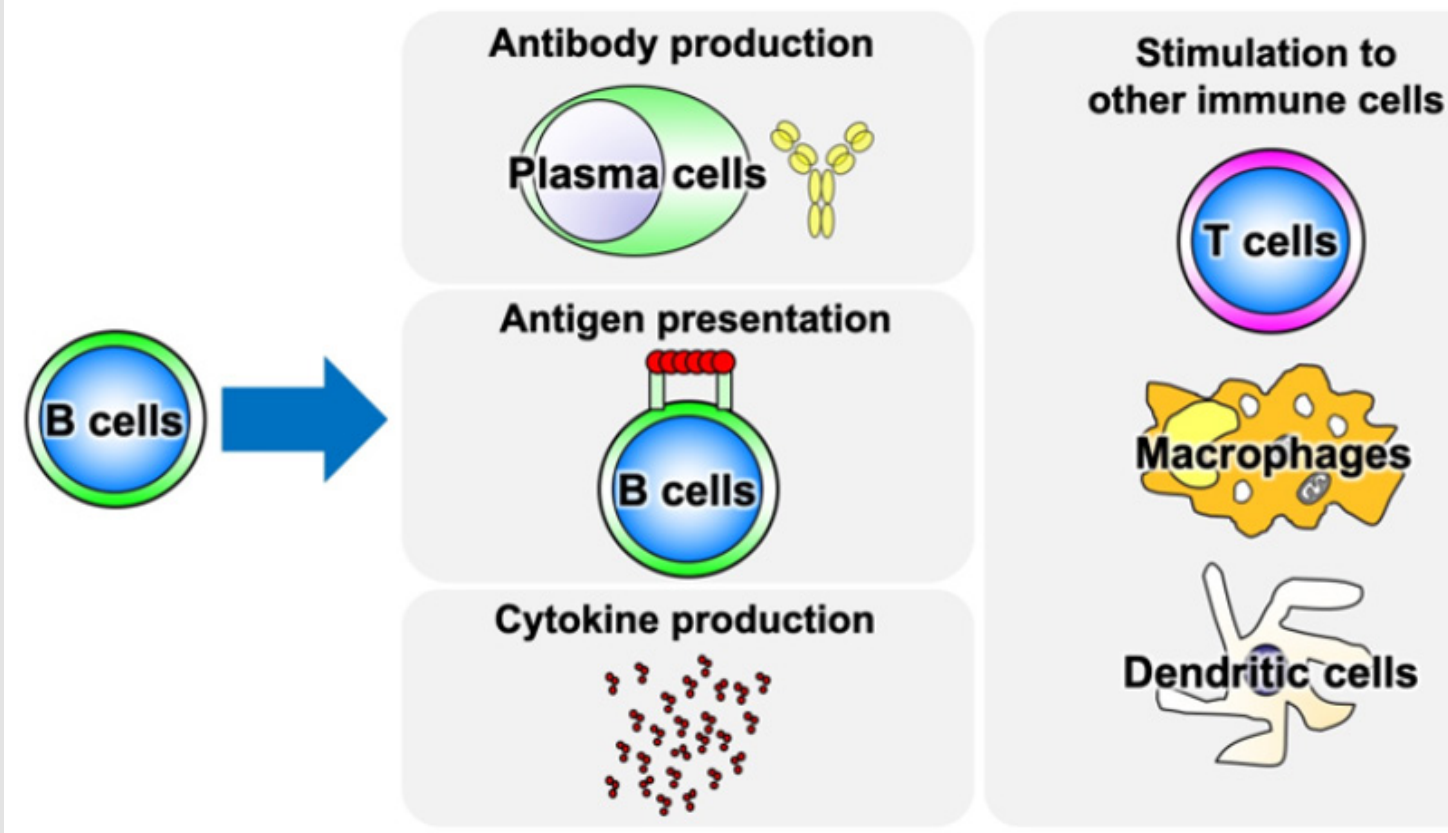

Figure 1: The diverse roles of B cells.

Note: B cells have antigen-presenting and cytokine-producing abilities in addition to antibody production. Through these functions, B cells directly or indirectly stimulate T cells, macrophages, and dendritic cells to induce activation and differentiation.

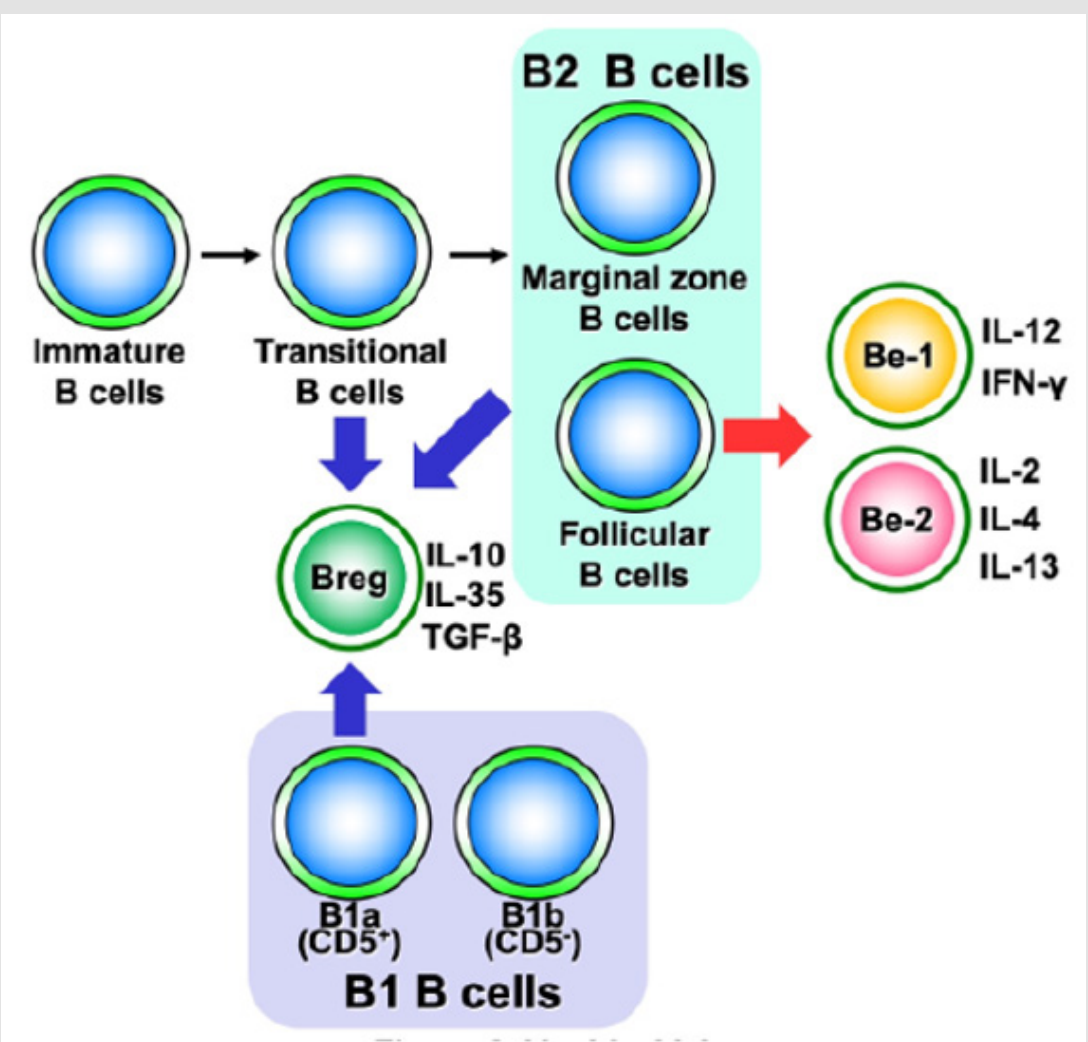

Figure 2: Relationship between B cell differentiation and cytokine-producing B cell subsets.

Note: Cytokine-producing B cells are mainly classified into Be-1, Be-2, and Breg cells. Be-1 and Be-2 cells are found in follicular B cells, wherein Breg cells are present in transitional B cells, B2 cells, and B1 cells. 


\section{Be cells}

Be-1 cells produce Th1-type cytokines such as interferon (IFN)- $\gamma$ and interleukin (IL)-12 upon stimulation by Th1 cells or Th1-type response-inducing antigens [6]. Be-1 cells were identified in an infection experiment that induced a Th1-type response in mice. On the other hand, Be- 2 cells were identified in a model of parasitic infection [6]. Be-2 cells have been shown to produce IL-2, IL-4, IL-12, and other Th2-type cytokines in response to Th2 cell stimulation, Th2-type antigen stimulation, and allergic reactions. B cells are classified into B1 and B2 cells, depending on their developmental origin and distribution. B1 cells originate in the liver during embryonic period and are distributed in mucosal tissues, abdominal cavity, and thoracic cavity. B2 cells come from the bone marrow. Furthermore, based on their distribution in lymphoid tissues, B2 cells are divided into marginal zone B cells and follicular B cells. Marginal zone B cells are important for innate immunity, while follicular B cells are crucial for antigenspecific acquired immunity. Recent studies have shown that Be cells are present in follicular B cells [7]. Thus, it has been suggested that Be cells may be intimately involved in autoimmune diseases through autoantigen-specific responses. However, to the best of our knowledge, no studies have directly examined autoantigen-reactive Be cells in patients with autoimmune diseases. This is because the amount of cytokines produced by B cells is small compared to dendritic cells, macrophages, and $\mathrm{T}$ cells, and in addition, there are only a few disease specific autoantigen-reactive B cells in the patient's body, making direct studies difficult. Therefore, there is not much knowledge available on the relationship between Be cells and autoimmune diseases.

\section{Breg Cells}

Breg cells are thought to exert an immunosuppressive function by producing inhibitory cytokines such as IL-10, IL-35, and transforming growth factor- $\beta[8,9]$. Unlike Be cells, which are relatively confined to follicular B cells, Breg cells are present in various B cell subpopulations, including B1a cells showing CD5 positivity in B1 cells, transitional B cells, follicular B cells, and marginal zone B cells. The function of Breg cells has so far been mainly defined by their ability to produce anti-inflammatory cytokines, but several studies in mice have suggested that the role of Breg cells depends on which fraction they belong to [10,11]. These studies have shown that Breg cells in the B1a fraction suppress the excessive immune response to innate immunity during the neonatal phase, and that Breg cells in marginal zone B cell or transitional B cell fractions, which are B2 cells, suppress the autoimmune response during the adult phase. In humans, Breg cells with traits of marginal zone B cells that strongly express CD1d or transitional B cells that express both CD24 and CD38 are considered to be responsible for the suppression of activity in several autoimmune diseases [12]. In addition, since adoptive transfer of Breg cells markedly suppresses the symptoms of many autoimmune disease models, its use as a treatment for humans is notable [13].

\section{Conclusion}

In addition to antibody production and antigen presentation, B cells have been shown to play a variety of roles through cytokine production. However, the autoantigen-specific response, which is the main feature of B cells in autoimmune diseases, has not been fully studied in autoimmune disease patients. The reasons for this are the low number of disease specific autoantigen-reactive B cells in patients and the low amount of cytokines produced by B cells, as mentioned above. To solve this conundrum, we have developed a unique system using microfluidics to measure very small amounts of cytokines at the ag-fg/ml levels through collaborative research in medical and engineering [14-17]. Studies using this system have shown that IL-10-producing B cell population exist even among autoantigen-reactive B cells, suggesting that not all autoantigenreactive B cells are pathogenic [14]. In fact, B cell depletion therapy has been shown to be effective in several autoimmune diseases, but there is still room for improvement in the general removal of all B cells, as adverse events such as infections have sometimes been observed. However, the detailed functional analysis of B cells ultimately needs to be performed in a single cell, because not only the diverse subsets of $B$ cells exist, but also the differences between individual B cells according to the different B cell receptors [18]. It is expected that further innovative B cell studies, such as single B cell analysis at protein levels, will clarify the relationship between $B$ cell subsets in the context of cytokine production and autoantigenreactive B cells, which will lead to the discovery of novel therapeutic targets.

\section{Conflict of Interest}

The authors have declared that no conflict of interest exists.

\section{References}

1. Lipsky PE (2001) Systemic lupus erythematosus: an autoimmune disease of B cell hyperactivity. Nat Immunol 2(9): 764-766.

2. Pollmann R, Walter E, Schmidt T, Waschke J, Hertl M, et al. (2019) Identification of Autoreactive B Cell Subpopulations in Peripheral Blood of Autoimmune Patients With Pemphigus Vulgaris. Front Immunol 10: 1375 .

3. Schioppo T, Ingegnoli $\mathrm{F}$ (2017) Current perspective on rituximab in rheumatic diseases. Drug Des Devel Ther 11: 2891-2904.

4. Fu YX, Huang G, Wang Y, Chaplin DD (1998) B lymphocytes induce the formation of follicular dendritic cell clusters in a lymphotoxin alphadependent fashion. J Exp Med 187(7): 1009-1018.

5. Lund FE (2008) Cytokine-producing B lymphocytes-key regulators of immunity. Curr Opin Immunol 20(3): 332-338.

6. Harris DP, Haynes L, Sayles PC, Duso DK, Eaton SM, et al. (2000) Reciprocal regulation of polarized cytokine production by effector $B$ and T cells. Nat Immunol 1(6): 475-482.

7. Cariappa A, Boboila C, Moran ST, Liu H, Shi HN, et al. (2007) The recirculating $\mathrm{B}$ cell pool contains two functionally distinct, long-lived, posttransitional, follicular B cell populations. J Immunol 179(4): 22702281. 
8. Yanaba K, Bouaziz JD, Haas KM, Poe JC, Fujimoto M, et al. (2008) A regulatory B cell subset with a unique CD1dhiCD5+ phenotype controls T cell-dependent inflammatory responses. Immunity 28(5): 639-650.

9. Rosser EC, Mauri C (2015) Regulatory B cells: origin, phenotype, and function. Immunity 42(4): 607-612.

10. Zhang X, Deriaud E, Jiao X, Braun D, Leclerc C, et al. (2007) Type I interferons protect neonates from acute inflammation through interleukin 10-producing B cells. J Exp Med 204(5): 1107-1118.

11. Evans JG, Chavez Rueda KA, Eddaoudi A, Meyer Bahlburg A, Rawlings DJ, et al. (2007) Novel suppressive function of transitional 2 B cells in experimental arthritis. J Immunol 178(12): 7868-7878.

12. Mauri C, Menon M (2017) Human regulatory B cells in health and disease: therapeutic potential. J Clin Invest 127(3): 772-779.

13. Yoshizaki A, Miyagaki T, DiLillo DJ, Matsushita T, Horikawa M, et al. (2012) Regulatory B cells control T-cell autoimmunity through IL-21dependent cognate interactions. Nature 491(7423): 264-268.

ISSN: 2574-1241

DOI: $10.26717 /$ BJSTR.2020.28.004638

Ayumi Yoshizaki. Biomed J Sci \& Tech Res

(C) This work is licensed under Creative Commons Attribution 4.0 License

Submission Link: https://biomedres.us/submit-manuscript.php
14. Fukasawa T, Yoshizaki A, Ebata S, Nakamura K, Saigusa R, et al. (2017) Contribution of Soluble Forms of Programmed Death 1 and Programmed Death Ligand 2 to Disease Severity and Progression in Systemic Sclerosis. Arthritis \& rheumatology 69(9): 1879-1890.

15. Lin L, Mawatari K, Morikawa K, Pihosh Y, Yoshizaki A, et al. (2017) Micro/extended-nano sampling interface from a living single cell. Analyst 142(10): 1689-1696.

16. Nakao T, Kazoe Y, Mori E, Morikawa K, Fukasawa T, et al. (2019) Cytokine analysis on a countable number of molecules from living single cells on nanofluidic devices. Analyst 144(24): 7200-7208.

17. Numajiri H, Yoshizaki A, Fukasawa T, Ebata S, Nakamura K, et al. (2018) Rapid alteration of serum interleukin- 6 levels may predict the reactivity of i.v. cyclophosphamide pulse therapy in systemic sclerosis-associated interstitial lung disease. J Dermatol 45(10): 1221-1224.

18. Liu W, Meckel T, Tolar P, Sohn HW, Pierce SK (2010) Antigen affinity discrimination is an intrinsic function of the B cell receptor. J Exp Med 207(5): 1095-1111.

$\begin{array}{ll}\text { BIOMEDICAL } & \text { Assets of Publishing with us } \\ \text { RESEARCHES } & \text { - Global archiving of articles } \\ & \text { - Immediate, unrestricted online access } \\ & \text { - Rigorous Peer Review Process } \\ & \end{array}$

